\title{
Admissibility of Diagonal State-Delayed Systems with a One-Dimensional Input Space
}

\author{
Jonathan R. Partington ${ }^{1} \cdot$ Radosław Zawiski ${ }^{2}$
}

Received: 11 May 2018 / Accepted: 5 March 2019 / Published online: 18 March 2019

(c) The Author(s) 2019

\begin{abstract}
In this paper we investigate admissibility of the control operator $B$ in a Hilbert space state-delayed dynamical system setting of the form $\dot{z}(t)=A z(t-\tau)+B u(t)$, where $A$ generates a diagonal semigroup and $u$ is a scalar input function. Our approach is based on the Laplace embedding between $L^{2}$ and the Hardy space. The sufficient conditions for infinite-time admissibility are stated in terms of eigenvalues of the generator and in terms of the control operator itself.
\end{abstract}

Keywords Admissibility $\cdot$ State delay $\cdot$ Diagonal system $\cdot$ Reciprocal system

\section{Introduction}

In this article we analyse dynamical system with delay in the state variable from the perspective of admissibility of the control operator. Thus the object of our interest is an abstract dynamical system

$$
\left\{\begin{array}{l}
\dot{z}(t)=A z(t-\tau)+B u(t) \\
z(0)=z_{0}
\end{array}\right.
$$

Communicated by Sanne ter Horst, Dmitry Kaliuzhnyi-Verbovetskyi, Izchak Lewkowicz and Daniel Alpay.

The research presented here was done when the second author was a Marie Curie Research Fellow at the School of Mathematics, University of Leeds, UK.

$凶$ Radosław Zawiski

radoslaw.zawiski@polsl.pl

1 School of Mathematics, University of Leeds, Leeds LS2 9JT, UK

2 Institute of Automatic Control, Silesian University of Technology, Akademicka 16, 44-100 Gliwice, Poland 
where, in general, $A: D(A) \subset X \rightarrow X$ is the infinitesimal generator of a $C_{0^{-}}$ semigroup $(T(t))_{t \geq 0}$ on $X$. The Hilbert space $X$ possesses a sequence of normalized eigenvectors $\left(\phi_{k}\right)_{k \in \mathbb{N}}$ forming a Riesz basis, with associated eigenvalues $\left(\lambda_{k}\right)_{k \in \mathbb{N}}$. The input function is $u \in L^{2}(0, \infty ; \mathbb{C}), B$ is the control operator and $0<\tau<\infty$ is a delay.

Infinite-time admissibility of $B$ in the undelayed case of (1) is well analysed and the necessary and sufficient conditions for it were given using e.g. Carleson measures. In particular, the link between Carleson measures and infinite-time admissibility was studied in $[10,11,23]$. Those results were extended to normal semigroups [24], then generalized to the case when $u \in L^{2}\left(0, \infty ; t^{\alpha} d t\right)$ for $\alpha \in(-1,0)$ in [25] and further to the case $u \in L^{2}(0, \infty ; w(t) d t)$ in $[13,14]$. For a thorough presentation of admissibility results, not restricted to diagonal systems, for the undelayed case we refer the reader to [12] and a rich list of references therein.

The results in [9] and [5] form a basis for considerations in [2] in terms of developing a correct setting in which we conduct the admissibility analysis for state-delayed diagonal systems. The same setting is used by us for the admissibility analysis in a more general case when (1) takes a form of the so-called retarded equation, where we assume only a contraction property of the undelayed semigroup $(T(t))_{t \geq 0}$ (full details will be published elsewhere [26])

Section 2 contains the necessary background results, leading to the main results in Sect. 3. An example is given in Sect. 4, and some conclusions are given in Sect. 5.

\section{Preliminaries}

Apart from definitions introduced in the previous section throughout this paper we use the following Sobolev spaces (see [17] for vector valued functions or [7, Chapter 5] for functionals): $W^{1,2}(J, X):=\left\{f \in L^{2}(J, X): \frac{d}{d t} f(t) \in L^{2}(J, X)\right\}, W_{c}^{1,2}(J, X):=$ $\left\{f \in W^{1,2}(J, X): f\right.$ has compact support $\}$ and $W_{0}^{1,2}(J, X):=\left\{f \in W^{1,2}(J, X)\right.$ : $f(\partial J)=0\}$, where $J$ is an interval.

For any $\alpha \in \mathbb{R}$ we denote $\mathbb{C}_{\alpha}:=\{s \in \mathbb{C}:$ Res $>\alpha\}$ with an exception for two special cases, namely $\mathbb{C}_{+}:=\{s \in \mathbb{C}: \operatorname{Re} s>0\}$ and $\mathbb{C}_{-}:=\{s \in \mathbb{C}: \operatorname{Re} s<0\}$.

The Hardy space $H^{2}\left(\mathbb{C}_{+}\right)$consists of all analytic functions $f: \mathbb{C}_{+} \rightarrow \mathbb{C}$ for which

$$
\sup _{\alpha>0} \int_{-\infty}^{\infty}|f(\alpha+i \omega)|^{2} d \omega<\infty
$$

If $f \in H^{2}\left(\mathbb{C}_{+}\right)$then for almost every $\omega \in \mathbb{R}$ the limit

$$
f^{*}(i \omega)=\lim _{\alpha \downarrow 0} f(\alpha+i \omega)
$$

exists and defines a function $f^{*} \in L^{2}(i \mathbb{R})$ called the boundary trace of $f$. Using boundary traces $H^{2}\left(\mathbb{C}_{+}\right)$is made into a Hilbert space with the inner product defined as 


$$
\langle f, g\rangle_{H^{2}\left(\mathbb{C}_{+}\right)}:=\left\langle f^{*}, g^{*}\right\rangle_{L^{2}(i \mathbb{R})}:=\frac{1}{2 \pi} \int_{-\infty}^{+\infty} f^{*}(i \omega) \bar{g}^{*}(i \omega) d \omega
$$

for every $f, g \in H^{2}\left(\mathbb{C}_{+}\right)$. For more information about Hardy spaces see $[8,18]$ or [16]. We also make use of the following

Theorem 2.1 (Paley-Wiener) Let $Y$ be a Hilbert space. Then the Laplace transform $\mathcal{L}: L^{2}(0, \infty ; Y) \rightarrow H^{2}\left(\mathbb{C}_{+} ; Y\right)$ is an isometric isomorphism.

For a detailed proof of Theorem 2.1 see [20, Chapter 19] for the scalar version or [1, Theorem 1.8.3] for the vector-valued one.

\subsection{The Delayed Equation Setting}

For details of the setting in which we consider a state-delayed diagonal system see [6, Chapter VI.6] and [2, Chapter 3.1]. Consider a function $z:[-\tau, \infty) \rightarrow X$. For each $t \geq 0$ we call the function $z_{t}:[-\tau, 0] \rightarrow X, z_{t}(\sigma):=z(t+\sigma)$, a history segment with respect to $t \geq 0$. With history segments we consider a function called the history function of $z$, that is $h_{z}:[0, \infty) \rightarrow L^{2}(-\tau, 0 ; X), h_{z}(t):=z_{t}$. In [2, Lemma 3.4] we find the following

Proposition 2.2 Let $1 \leq p<\infty$ and $z:[-\tau, \infty) \rightarrow X$ be a function which belongs to $W_{l o c}^{1, p}(-\tau, \infty ; X)$. Then the history function $h_{z}: t \rightarrow z_{t}$ of $z$ is continuously differentiable from $\mathbb{R}_{+}$into $L^{p}(-\tau, 0 ; X)$ with derivative $\frac{\partial}{\partial t} h_{z}(t)=\frac{\partial}{\partial \sigma} z_{t}$.

Define the Cartesian product $\mathcal{X}:=X \times L^{2}(-\tau, 0 ; X)$ with an inner product

$$
\left\langle\left(\begin{array}{l}
x \\
f
\end{array}\right),\left(\begin{array}{l}
y \\
g
\end{array}\right)\right\rangle_{\mathcal{X}}:=\langle x, y\rangle_{X}+\langle f, g\rangle_{L^{2}(-\tau, 0 ; \mathbb{C})} .
$$

Then $\mathcal{X}$ becomes a Hilbert space $(\mathcal{X},\|\cdot\| \mathcal{X})$ with the norm $\left\|\left(\begin{array}{l}x \\ f\end{array}\right)\right\|_{\mathcal{X}}^{2}=\|x\|_{X}^{2}+\|f\|_{L^{2}}^{2}$. Consider a linear, autonomous delay differential equation of the form

$$
\left\{\begin{array}{l}
\dot{z}(t)=A z(t)+\Psi z_{t} \\
z(0)=x \\
z_{0}=f
\end{array}\right.
$$

where $\Psi \in \mathcal{L}\left(W^{1,2}(-\tau, 0 ; X), X\right)$ is a delay operator, the pair $x \in D(A)$ and $f \in$ $L^{2}(-\tau, 0 ; X)$ forms an initial condition. Due to Proposition 2.2 equation (6) may be written as an abstract Cauchy problem

$$
\left\{\begin{array}{l}
\dot{v}(t)=\mathcal{A} v(t) \\
v(0)=\left(\begin{array}{l}
x \\
f
\end{array}\right)
\end{array}\right.
$$

where $v: t \rightarrow\left(\begin{array}{c}z(t) \\ z_{t}\end{array}\right) \in \mathcal{X}$ and $\mathcal{A}$ is an operator on $\mathcal{X}$ defined as

$$
\mathcal{A}:=\left(\begin{array}{cc}
A & \Psi \\
0 & \frac{d}{d \sigma}
\end{array}\right)
$$


with domain

$$
D(\mathcal{A}):=\left\{\left(\begin{array}{l}
x \\
f
\end{array}\right) \in D(A) \times W^{1,2}(-\tau, 0 ; X): f(0)=x\right\} .
$$

The operator $(\mathcal{A}, D(\mathcal{A}))$ is closed and densely defined on $\mathcal{X}$ [2, Lemma 3.6]. Let $\mathcal{A}=\mathcal{A}_{0}+\mathcal{A}_{\Psi}$, where

$$
\mathcal{A}_{0}:=\left(\begin{array}{cc}
A & 0 \\
0 & \frac{d}{d \sigma}
\end{array}\right), \quad D\left(\mathcal{A}_{0}\right)=D(\mathcal{A})
$$

and

$$
\mathcal{A}_{\Psi}:=\left(\begin{array}{ll}
0 & \Psi \\
0 & 0
\end{array}\right) \in \mathcal{L}\left(X \times W^{1,2}(-\tau, 0 ; X), \mathcal{X}\right) .
$$

We will need the following for the Miyadera-Voigt Perturbation Theorem and a description of admissibility.

Definition 2.3 Let $\beta \in \rho(A)$ and denote $\left(X_{1},\|\cdot\|_{1}\right):=\left(D(A),\|\cdot\|_{1}\right)$ with $\|\cdot\|_{1}:=$ $\|(\beta I-A) x\|(x \in D(A))$.

Similarly, we set $\|x\|_{-1}:=\left\|(\beta I-A)^{-1} x\right\|(x \in X)$. Then the space $\left(X_{-1},\|\cdot\|_{-1}\right)$ denotes the completion of $X$ under the norm $\|\cdot\|_{-1}$. For $t \geq 0$ we define $T_{-1}(t)$ as the continuous extension of $T(t)$ to the space $\left(X_{-1},\|\cdot\|_{-1}\right)$.

In the sequel, much of our reasoning is justified by the following Proposition, to which we do not refer directly but include here for the reader's convenience.

Proposition 2.4 With notation of Definition 2.3 we have the following

(i) The spaces $\left(X_{1},\|\cdot\|_{1}\right)$ and $\left(X_{-1},\|\cdot\|_{-1}\right)$ are independent of the choice of $\beta \in$ $\rho(A)$.

(ii) $\left(T_{1}(t)\right)_{t \geq 0}$ is a strongly continuous semigroup on the Banach space $\left(X_{1},\|\cdot\|_{1}\right)$ and we have $\left\|T_{1}(t)\right\|_{1}=\|T(t)\|$ for all $t \geq 0$.

(iii) $\left(T_{-1}(t)\right)_{t \geq 0}$ is a strongly continuous semigroup on the Banach space $\left(X_{-1},\|\cdot\|_{-1}\right)$ and we have $\left\|T_{-1}(t)\right\|_{-1}=\|T(t)\|$ for all $t \geq 0$.

See [6, Chapter II.5] or [21, Chapter 2.10] for more details on these elements. A sufficient condition for $P \in \mathcal{L}\left(X_{1}, X\right)$ to be a perturbation of Miyadera-Voigt class, and hence implying that $A+P$ is a generator on $X$, takes the form of [6, Corollary III.3.16]

Proposition 2.5 Let $(A, D(A))$ be the generator of a strongly continuous semigroup $(T(t))_{t \geq 0}$ on a Banach space $X$ and let $P \in \mathcal{L}\left(X_{1}, X\right)$ be a perturbation which satisfies

$$
\int_{0}^{t_{0}}\|P T(r) x\| d r \leq q\|x\| \quad \forall x \in D(A)
$$

for some $t_{0}>0$ and $0 \leq q<1$. Then the sum $A+P$ with domain $D(A+P):=D(A)$ generates a strongly continuous semigroup $(S(t))_{t \geq 0}$ on $X$. 
To describe the resolvent of $(\mathcal{A}, D(\mathcal{A}))$, let us introduce the notation

$$
A_{0}:=\frac{d}{d \sigma}, \quad D\left(A_{0}\right)=\left\{z \in W^{1,2}(-\tau, 0 ; X): z(0)=0\right\}
$$

for the generator of the nilpotent left shift semigroup on $L^{p}(-\tau, 0 ; X)$. For $s \in \mathbb{C}$ define $\epsilon_{s}:[-\tau, 0] \rightarrow \mathbb{C}, \epsilon_{s}(\sigma):=e^{s \sigma}$. Define also $\Psi_{s} \in \mathcal{L}(D(A), X), \Psi_{s} x:=$ $\Psi\left(\epsilon_{S}(\cdot) x\right)$. Then [2, Proposition 3.19] provides

Proposition 2.6 For $s \in \mathbb{C}$ and for all $1 \leq p<\infty$ we have

$$
s \in \rho(\mathcal{A}) \text { if and only if } s \in \rho\left(A+\Psi_{s}\right) .
$$

Moreover, for $s \in \rho(\mathcal{A})$ the resolvent operator $R(s, \mathcal{A})$ is given by

$$
R(s, \mathcal{A})=\left(\begin{array}{ll}
R\left(s, A+\Psi_{s}\right) & R\left(s, A+\Psi_{s}\right) \Psi R\left(s, A_{0}\right) \\
\epsilon_{s} R\left(s, A+\Psi_{s}\right) & \left(\epsilon_{s} R\left(s, A+\Psi_{s}\right) \Psi+I\right) R\left(s, A_{0}\right)
\end{array}\right)
$$

\subsection{The Admissibility Problem}

The basic object in the formulation of admissibility problem is a linear system and its mild solution

$$
\frac{d}{d t} x(t)=A x(t)+B u(t) ; \quad x(t)=T(t) x_{0}+\int_{0}^{t} T(t-s) B u(s) d s,
$$

where $x:[0, \infty) \rightarrow X, u \in V$ where $V$ is a space of measurable functions from $[0, \infty)$ to $U$ and $B$ is a control operator; $x_{0} \in X$ is an initial state.

In many practical examples the control operator $B$ is unbounded, hence (14) is viewed on an extrapolation space $X_{-1} \supset X$ where $B \in \mathcal{L}\left(U, X_{-1}\right)$. To ensure that the state $x(t)$ lies in $X$ it is sufficient that $\int_{0}^{t} T_{-1}(t-s) B u(s) d s \in X$ for all inputs $u \in V$. Put differently, we have

Definition 2.7 The control operator $B \in \mathcal{L}\left(U, X_{-1}\right)$ is said to be finite-time admissible for a semigroup $(T(t))_{t \geq 0}$ on a Hilbert space $X$ if for each $\tau>0$ there is a constant $c(\tau)$ such that the condition

$$
\left\|\int_{0}^{\tau} T_{-1}(\tau-s) B u(s) d s\right\|_{X} \leq c(\tau)\|u\|_{V}
$$

holds for all inputs $u$, and an infinite-time admissible if the condition (15) holds for all $\tau>0$ with $c(\tau)$ uniformly bounded.

In the sequel, we denote the restriction (extension) of $T(t)$ described in Definition 2.3 by the same symbol $T(t)$, since this is unlikely to lead to confusions. 


\section{Diagonal Non-autonomous Delay Systems}

We begin with an analysis of (1) in a more concrete setting. Consider the system

$$
\left\{\begin{array}{l}
\dot{z}(t)=A z(t-\tau)+B u(t) \\
z(0)=x \\
z_{0}=f
\end{array}\right.
$$

where the state space is $X:=l^{2}(\mathbb{C})$, the control function $u \in L^{2}(0, \infty ; \mathbb{C})$ and $\left(\lambda_{k}\right)_{k \in \mathbb{N}}$ is a sequence in $\mathbb{C}$ such that

$$
\lambda_{k} \in \mathbb{C}_{-} \quad \forall k \in \mathbb{N}
$$

The semigroup generator $(A, D(A))$ is defined by

$$
(A z)_{k}:=\lambda_{k} z_{k}, \quad D(A):=\left\{z \in l^{2}(\mathbb{C}): \sum_{k \in \mathbb{N}}\left(1+\left|\lambda_{k}\right|^{2}\right)\left|z_{k}\right|^{2}<\infty\right\} .
$$

As the space $X_{1}$ we take $\left(D(A),\|\cdot\|_{g r}\right)$, where the graph norm is equivalent to

$$
\|z\|_{1}^{2}=\sum_{k \in \mathbb{N}}\left(1+\left|\lambda_{k}\right|^{2}\right)\left|z_{k}\right|^{2}
$$

The adjoint generator $A^{*}$ is represented in the same way, with the sequence $\left(\bar{\lambda}_{k}\right)_{k \in \mathbb{N}}$ in place of $\left(\lambda_{k}\right)_{k \in \mathbb{N}}$. This gives $D\left(A^{*}\right)=D(A)$. The space $X_{-1}$ consists of all sequences $z=\left(z_{k}\right)_{k \in \mathbb{N}} \in \mathbb{C}^{\mathbb{N}}$ for which

$$
\sum_{k \in \mathbb{N}} \frac{\left|z_{k}\right|^{2}}{1+\left|\lambda_{k}\right|^{2}}<\infty
$$

and the square root of the above series gives an equivalent norm on $X_{-1}$. The space $X_{-1}$ is the same as $X_{-1}^{d}$, where the latter one is the equivalent of $X_{-1}$ should the construction in Definition 2.3 be based on $A^{*}$ instead of $A$.

Note also that the operator $B \in \mathcal{L}\left(\mathbb{C}, X_{-1}\right)$ is represented by the sequence $\left(b_{k}\right)_{k \in \mathbb{N}} \in \mathbb{C}^{\mathbb{N}}$, as $\mathcal{L}\left(\mathbb{C}, X_{-1}\right)$ can be identified with $X_{-1}$.

The above is the standard setting for diagonal systems; we refer the reader to [21, Chapters 2.6 and 5.3] for more details.

Remark 3.1 Although we restrict ourselves to contraction semigroups, this does not lead to loss of generality due to the semigroup rescaling property. That is when $A$ does not generate a contraction semigroup, we may replace it with a shifted version $A-\alpha I$ for a sufficiently large $\alpha>0$. This does not change the admissibility of control operator for the rescaled semigroup, but may change the infinite time admissibility. 


\subsection{Analysis of a Single Component}

Let us now focus on the $k$-th component of (16), that is

$$
\left\{\begin{array}{l}
\dot{z}_{k}(t)=\lambda_{k} z_{k}(t-\tau)+b_{k} u(t) \\
z_{k}(0)=x_{k} \\
z_{0_{k}}=f_{k}
\end{array}\right.
$$

where $\lambda_{k}, b_{k}, x_{k} \in \mathbb{C}, f_{k}:=\left\langle f, l_{k}\right\rangle_{L^{2}(-\tau, 0 ; X)} l_{k}$ with $l_{k}$ being the $k$-th component of the standard orthonormal basis in $L^{2}(-\tau, 0 ; X)$.

For the sake of clarity of notation, let us now until the end of this subsection drop the subscript $k$ and rewrite (19) in the form

$$
\left\{\begin{array}{l}
\dot{z}(t)=\Psi z_{t}+b u(t) \\
z(0)=x \\
z_{0}=f
\end{array}\right.
$$

where the delay operator $\Psi \in \mathcal{L}\left(W^{1,2}(-\tau, 0 ; \mathbb{C}), \mathbb{C}\right)$ is defined as

$$
\Psi(f):=\lambda f(-\tau) \quad \forall f \in W^{1,2}(-\tau, 0 ; \mathbb{C}) .
$$

Observe that, without the input function $b u \in L^{2}(0, \infty ; \mathbb{C})$, system $(20)$ is a simplified form of (6). As for such, we can apply the procedure described in the Preliminaries section and represent it as an abstract Cauchy problem of the form (7). For that purpose note that

$$
\mathcal{X}:=\mathbb{C} \times L^{2}(-\tau, 0 ; \mathbb{C})
$$

with an inner product

$$
\left\langle\left(\begin{array}{l}
x \\
f
\end{array}\right),\left(\begin{array}{l}
y \\
g
\end{array}\right)\right\rangle_{\mathcal{X}}:=x \bar{y}+\langle f, g\rangle_{L^{2}(-\tau, 0 ; \mathbb{C})} \forall\left(\begin{array}{l}
x \\
f
\end{array}\right),\left(\begin{array}{l}
y \\
g
\end{array}\right) \in \mathcal{X} .
$$

What follows is the non-autonomous Cauchy problem describing the dynamics of the $k$-th component

$$
\left\{\begin{array}{l}
\dot{v}(t)=\mathcal{A} v(t)+\mathcal{B} u(t) \\
v(0)=\left(\begin{array}{l}
x \\
f
\end{array}\right)
\end{array}\right.
$$

where $v: t \rightarrow\left(\begin{array}{c}z(t) \\ z_{t}\end{array}\right) \in \mathcal{X}$ and $\mathcal{A}$ is an operator on $\mathcal{X}$ defined as

$$
\mathcal{A}:=\left(\begin{array}{cc}
0 & \Psi \\
0 & \frac{d}{d \sigma}
\end{array}\right)
$$

with domain

$$
D(\mathcal{A}):=\left\{\left(\begin{array}{l}
x \\
f
\end{array}\right) \in \mathbb{C} \times W^{1,2}(-\tau, 0 ; \mathbb{C}): f(0)=x\right\},
$$


and $\mathcal{B}:=\left(\begin{array}{l}b \\ 0\end{array}\right) \in \mathcal{L}\left(\mathbb{C}, \mathcal{X}_{-1}\right)$. To state explicitly how the $\mathcal{X}_{-1}$ space looks like we use again (25) and (26) as well as Proposition 3.1 from [26]. As a result,

$$
\mathcal{X}_{-1}=\mathbb{C} \times W^{-1,2}(-\tau, 0 ; \mathbb{C}),
$$

where $W^{-1,2}(-\tau, 0 ; \mathbb{C})$ is the dual to $W_{0}^{1,2}(-\tau, 0 ; \mathbb{C})$ with respect to the pivot space $L^{2}(-\tau, 0 ; \mathbb{C})$. The generator $\mathcal{A}$ may again be represented as $\mathcal{A}=\mathcal{A}_{0}+\mathcal{A}_{\Psi}$, where

$$
\mathcal{A}_{0}:=\left(\begin{array}{cc}
0 & 0 \\
0 & \frac{d}{d \sigma}
\end{array}\right), \quad D\left(\mathcal{A}_{0}\right)=D(\mathcal{A})
$$

and

$$
\mathcal{A}_{\Psi}:=\left(\begin{array}{ll}
0 & \Psi \\
0 & 0
\end{array}\right) \in \mathcal{L}\left(\mathbb{C} \times W^{1,2}(-\tau, 0 ; \mathbb{C}), \mathcal{X}\right) .
$$

We have the following

Proposition 3.2 The abstract Cauchy problem (24) is well-posed.

Proof The delay operator $\Psi$ defined in (21) is an example of a much wider class of delay operators, with which condition (12) is satisfied and $(\mathcal{A}, D(\mathcal{A}))$ in (25) remains a generator of a strongly continuous semigroup $(\mathcal{T}(t))_{t \geq 0}$ on $\mathcal{X}$. See $[2$, Chapter 3.3 and Example 3.28] for details.

Due to Proposition 3.2 we can formally write the $\mathcal{X}_{-1}$-valued $k$-th component mild solution of (24)

$$
v(t)=\mathcal{T}(t) v(0)+\int_{0}^{t} \mathcal{T}(t-s) \mathcal{B} u(s) d s,
$$

where $\mathcal{T}(t) \in \mathcal{L}\left(\mathcal{X}_{-1}\right)$ and the control operator is again $\mathcal{B}=\left(\begin{array}{l}b \\ 0\end{array}\right) \in \mathcal{L}\left(\mathbb{C}, \mathcal{X}_{-1}\right)$. The following Proposition gives information concerning spectral properties and the resolvent operator $R(s, \mathcal{A})$.

Proposition 3.3 For $s \in \mathbb{C}$ and for all $1 \leq p<\infty$ there is

$$
s \in \rho(\mathcal{A}) \text { if and only if } s \in \rho\left(\Psi_{s}\right) .
$$

Moreover, for $s \in \rho(\mathcal{A})$ the resolvent operator $R(s, \mathcal{A})$ is given by

$$
R(s, \mathcal{A})=\left(\begin{array}{ll}
R\left(s, \Psi_{s}\right) & R\left(s, \Psi_{s}\right) \Psi R\left(s, A_{0}\right) \\
\epsilon_{s} R\left(s, \Psi_{s}\right) & \left(\epsilon_{s} R\left(s, \Psi_{s}\right) \Psi+I\right) R\left(s, A_{0}\right)
\end{array}\right),
$$

where $R\left(s, \Psi_{s}\right) \in \mathcal{L}(\mathbb{C})$,

$$
R\left(s, \Psi_{s}\right)=\frac{1}{s-\lambda \mathrm{e}^{-s \tau}} \quad \forall s \in \mathbb{C}_{|\lambda|}
$$

and $R\left(s, A_{0}\right) \in \mathcal{L}\left(L^{2}(-\tau, 0 ; \mathbb{C})\right)$,

$$
R\left(s, A_{0}\right) f(r)=\int_{r}^{0} \mathrm{e}^{s(r-t)} f(t) d t \quad r \in[-\tau, 0] \quad \forall s \in \mathbb{C}_{|\lambda|} .
$$


Proof 1. Condition (31) and the form of $R(s, \mathcal{A})$ in (32) follow directly from Proposition 2.6 and the form of $\mathcal{A}$ given in (25).

2. As is well known, for any Banach space $X$ and operator $A \in \mathcal{L}(X)$ the condition $s \in \sigma(A)$ implies $|s| \leq\|A\|$.

3. According to the definitions given before Proposition 2.6 in this case there is $\Psi_{s} \in$ $\mathcal{L}(\mathbb{C}), \Psi_{s} x:=\lambda \mathrm{e}^{-s \tau} x$ and $\left\|\Psi_{s}\right\|=|\lambda| \mathrm{e}^{-\operatorname{Re} s \tau}$. The equation $\left(\mu-\Psi_{s}\right) x=y$ has a unique solution $x \in \mathbb{C}$ for each $y \in \mathbb{C}$ if and only if $\mu \neq \lambda \mathrm{e}^{-s \tau}$. Thus $\sigma\left(\Psi_{S}\right)=\left\{\lambda \mathrm{e}^{-s \tau}\right\}$, and so

$$
\left\{s \in \mathbb{C}: s \in \sigma\left(\Psi_{s}\right)\right\} \subset\left\{s \in \mathbb{C}:|s| \leq|\lambda| \mathrm{e}^{-\operatorname{Re} s \tau}\right\} \subset\{s \in \mathbb{C}: \operatorname{Re} s \leq|\lambda|\} .
$$

Moreover, for $s \neq \lambda \mathrm{e}^{-s \tau}$ there is

$$
R\left(s, \Psi_{s}\right)=\frac{1}{s-\lambda \mathrm{e}^{-s \tau}}
$$

4. To complete the description of $R(s, \mathcal{A})$ consider now $f \in L^{2}(-\tau, 0 ; \mathbb{C}), g \in$ $W^{1,2}(-\tau, 0 ; \mathbb{C})$ and a formal differential equation

$$
\left(s I-A_{0}\right) g(r)=s g(r)-g^{\prime}(r)=f(r)
$$

with an initial condition imposed on $f$ in the form $f(0)=0$. Solving firstly a homogeneous equation and then using the method of variation of constants one obtains

$$
g(r)=\int_{r}^{0} \mathrm{e}^{s(r-t)} f(t) d t \quad \forall r \in[-\tau, 0] \forall s \in \mathbb{C}_{|\lambda|}
$$

(see also [15, p. 174, (6.6)]).

Denote now $R_{S} f(r):=g(r), r \in[-\tau, 0]$. Then, for every $f \in L^{2}(-\tau, 0 ; \mathbb{C})$ there is $\left(s I-A_{0}\right) R_{S} f(r)=f(r)$ and $R_{S}: L^{2}(-\tau, 0 ; \mathbb{C}) \rightarrow D\left(A_{0}\right)$ and $R_{S} \in$ $\mathcal{L}\left(L^{2}(-\tau, 0 ; \mathbb{C})\right)$. Let now $f \in D\left(A_{0}\right)$. A simple check shows that $R_{S}[(s I-$ $\left.\left.A_{0}\right) f(r)\right]=f(r), r \in[-\tau, 0]$. This means that $R_{S}$ is in fact a resolvent operator and we may write

$$
R\left(s, A_{0}\right) f(r)=R_{s} f(r)=\int_{r}^{0} \mathrm{e}^{s(r-t)} f(t) d t \quad \forall f \in L^{2}(-\tau, 0 ; \mathbb{C}) .
$$

Proposition 3.3 gives the form of the resolvent $R\left(s, \Psi_{s}\right)$ and assures that it is analytic on $\mathbb{C}_{|\lambda|}$. The value of $\lambda$ is valid for the given mode only and at this stage $|\lambda| \rightarrow \infty$ is allowed. Thus, as we will later require analyticity of $R\left(s, \Psi_{s}\right)$ in $\mathbb{C}_{+}$, a different approach is needed. For that reason we turn our attention to the complex coefficient exponential polynomial $P: \mathbb{C} \rightarrow \mathbb{C}$,

$$
P(s):=s-\lambda \mathrm{e}^{-s \tau},
$$


where $\lambda \in \mathbb{C}_{-}$is a complex coefficient and $\tau>0$.

The polynomial (36) in a more general form $A(s)+B(s) \mathrm{e}^{-s \tau}$ is known and widely studied in the theory of stability of finite dimensional dynamical systems-see e.g. [3, Chapter 13] or [19, Chapter 6] and references therein. The main difficulty in our case, in comparison to the references given above, is that the coefficients are complex. Nevertheless, we can use a modified Walton-Marshall approach [22] (or [19, Proposition 6.2.3]), as the following Proposition shows.

Remark 3.4 We take the principal argument of $\lambda$ to be $\operatorname{Arg}(\lambda) \in(-\pi, \pi]$.

We shall require the following subset of the complex plane, depending on $\tau>0$ :

$$
\Lambda_{\tau}:=\left\{\lambda \in \mathbb{C}_{-}: \operatorname{Arg}(\lambda) \in\left(-\pi,-\frac{\pi}{2}\right) \cup\left(\frac{\pi}{2}, \pi\right],|\lambda|<\frac{1}{\tau}\left(|\operatorname{Arg}(\lambda)|-\frac{\pi}{2}\right)\right\}
$$

Proposition 3.5 For a given $\tau>0$ and $\lambda \in \mathbb{C}_{-}$the condition $\lambda \in \Lambda_{\tau}$ is sufficient for the polynomial $P$ defined in (36) no to have right half-plane zeros (to be stable). In other words, all the solutions of the characteristic equation $P(s)=0$ belong to $\mathbb{C}_{-}$.

Proof 1. Consider initially the case when $\tau=0$. The polynomial $P$ has one root $s_{0}=\lambda$ and $s_{0} \in \mathbb{C}_{-}$.

2. Using Rouché's theorem (see e.g. [3, Theorem 12.2]) one can show that the zeros move continuously with $\tau$. As they start in $\mathbb{C}_{-}$it remains to establish when they cross the imaginary axis.

3. At the crossing of the imaginary axis there is $s=i \omega$ for some $\omega \in \mathbb{R}$ and the characteristic equation takes the form

$$
s-\lambda \mathrm{e}^{-s \tau}=0
$$

By point 2. we can treat (38) as an implicit function with $s=s(\tau)$ and check the direction in which zeros of it cross the imaginary axis by analysing the sgn $\operatorname{Re} \frac{d s}{d \tau}$ at $s=i \omega$. By calculating the implicit function derivative we obtain

$$
\frac{d s}{d \tau}=-\frac{s^{2}}{1+s \tau}
$$

As $s$ is purely imaginary and $\operatorname{sgn} \operatorname{Re} z=\operatorname{sgn} \operatorname{Re} z^{-1}$ we have

$$
\operatorname{sgn} \operatorname{Re} \frac{d s}{d \tau}>0
$$

and the zeros cross from the left to the right half-plane. What remains is to find for what $\tau$ this happens.

4. Taking the complex conjugate of (38) we obtain

$$
-s-\bar{\lambda} \mathrm{e}^{s \tau}=0
$$


Using both of the above equations to eliminate the exponential part we obtain $s^{2}=-|\lambda|^{2}$, hence $s= \pm i|\lambda|$. Choosing to work further with $s=i|\lambda|$ and substituting it into (38) we get

$$
-i \frac{\lambda}{|\lambda|}=\mathrm{e}^{i|\lambda| \tau}
$$

The corresponding equation for $s=-i|\lambda|$ is

$$
-i \frac{|\lambda|}{\lambda}=\mathrm{e}^{i|\lambda| \tau}
$$

which has the same form as (39), but replacing $\lambda$ by $\bar{\lambda}$.

5. Let now $\lambda=|\lambda| \mathrm{e}^{i \operatorname{Arg} \lambda}$ where $\operatorname{Arg}(\lambda) \in\left(-\pi,-\frac{\pi}{2}\right) \cup\left(\frac{\pi}{2}, \pi\right]$. This gives

$$
\operatorname{Arg} \lambda-\frac{\pi}{2} \in\left(-\frac{3 \pi}{2},-\pi\right) \cup\left(0, \frac{\pi}{2}\right]
$$

and from (39) we have

$$
0<|\lambda| \tau=\operatorname{Arg} \lambda-\frac{\pi}{2} \leq \frac{\pi}{2}
$$

The above brings us to an observation that if there exist $\lambda \in \mathbb{C}_{-}$and $\tau>0$ such that $s=i|\lambda|$ is a solution to (38) i.e. $s=\lambda \mathrm{e}^{-s \tau}$ then $\frac{\pi}{2}<\operatorname{Arg}(\lambda) \leq \pi$ and (41) is satisfied.

If we choose to work in point 4 . with $s=-i|\lambda|$ instead, then by symmetry we obtain that if there exist $\lambda \in \mathbb{C}_{-}$and $\tau>0$ such that $s=-i|\lambda|$ is a solution to $s=\lambda \mathrm{e}^{-s \tau}$ then $-\pi<\operatorname{Arg}(\lambda)<\frac{\pi}{2}$ and the equation $|\lambda| \tau=-\operatorname{Arg}(\lambda)-\frac{\pi}{2}$ is satisfied.

6. From the discussion in point 5. we draw two conclusions:

(a) given a diagonal system, with fixed $\left(\lambda_{k}\right)_{k \in \mathbb{N}}$, the delay $\tau$ assuring that each mode is stable satisfies

$$
\tau<\frac{1}{\left|\lambda_{k}\right|}\left(\left|\operatorname{Arg} \lambda_{k}\right|-\frac{\pi}{2}\right) \quad \forall k \in \mathbb{N},
$$

(b) given a delay $\tau$, the distribution of $\left(\lambda_{k}\right)_{k \in \mathbb{N}}$ for each mode to remain stable is

$$
\left|\lambda_{k}\right|<\frac{1}{\tau}\left(\left|\operatorname{Arg} \lambda_{k}\right|-\frac{\pi}{2}\right) \quad \forall k \in \mathbb{N} .
$$

Clearly $\left(\lambda_{k}\right)_{k \in \mathbb{N}} \subset \mathbb{C}_{-}$.

In geometrical terms Proposition 3.5 states that the stability of $P$ is preserved for given $\tau$ provided that we choose the $\lambda$ coefficients from the interior of the set that 
resembles an ellipse with apsides in 0 and $-\frac{\pi}{2 \tau}$, and which is elongated towards the latter one.

Referring now to Definition 2.7 and the mild solution of the $k$-th component (30) we introduce the forcing operator $\Phi_{\infty} \in \mathcal{L}\left(L^{2}(0, \infty ; \mathbb{C}), \mathcal{X}_{-1}\right)$,

$$
\Phi_{\infty}(u):=\int_{0}^{\infty} \mathcal{T}(t) \mathcal{B} u(t) d t
$$

where

$$
\mathcal{T}(t) \mathcal{B}=\left(\begin{array}{ll}
\mathcal{T}_{11}(t) & \mathcal{T}_{12}(t) \\
\mathcal{T}_{21}(t) & \mathcal{T}_{22}(t)
\end{array}\right)\left(\begin{array}{l}
b \\
0
\end{array}\right)=\left(\begin{array}{l}
\mathcal{T}_{11}(t) b \\
\mathcal{T}_{21}(t) b
\end{array}\right)
$$

Hence the forcing operator becomes

$$
\Phi_{\infty}(u)=\left(\begin{array}{c}
\int_{0}^{\infty} \mathcal{T}_{11}(t) b u(t) d t \\
\int_{0}^{\infty} \mathcal{T}_{21}(t) b u(t) d t
\end{array}\right) \in \mathcal{X}_{-1}
$$

We can represent formally a similar product with the resolvent operator $R(s, \mathcal{A})$ from (32), namely

$$
R(s, \mathcal{A}) \mathcal{B}=\left(\begin{array}{ll}
R_{11}(s) & R_{12}(s) \\
R_{21}(s) & R_{22}(s)
\end{array}\right)\left(\begin{array}{l}
b \\
0
\end{array}\right)=\frac{b}{s-\lambda \mathrm{e}^{-s \tau}}\left(\begin{array}{c}
1 \\
\epsilon_{s}
\end{array}\right)
$$

where the correspondence of sub-indices with elements of (32) is obvious and will be used from now on to shorten the notation.

The connection between the semigroup $\mathcal{T}(t)$ and the resolvent $R(s, \mathcal{A})$ is given by the Laplace transform (see e.g. [21, Chapter 2.3]) whenever the integral converges and

$$
R(s, \mathcal{A}) \mathcal{B}=\int_{0}^{\infty} \mathrm{e}^{-s r} \mathcal{T}(r) \mathcal{B} d r=b\left(\begin{array}{c}
\mathcal{L}\left(\mathcal{T}_{11}\right)(s) \\
\mathcal{L}\left(\mathcal{T}_{21}\right)(s)
\end{array}\right) \in \mathcal{L}\left(\mathbb{C}, \mathcal{X}_{-1}\right)
$$

We can now state the main theorem for the $k$-th component of the delay system (16), namely

Theorem 3.6 Let for the given delay $\tau$ the eigenvalue $\lambda$ satisfy $\lambda \in \Lambda_{\tau}$. Then the control operator $\mathcal{B}=\left(\begin{array}{l}b \\ 0\end{array}\right)$ for the system (24) is infinite-time admissible for every $u \in L^{2}(0, \infty ; \mathbb{C})$ and

$$
\left\|\Phi_{\infty} u\right\|_{\mathcal{X}}^{2} \leq|b|^{2}(1+\tau) \frac{1}{\pi}\left(\frac{2-\delta}{\delta|\lambda|}+\frac{2 \delta}{\left(1-m^{2}\right)|\lambda|}\right)\|u\|_{L^{2}(0, \infty ; \mathbb{C})}^{2},
$$

for some $\delta, m \in(0,1)$, which can be given explicitly in terms of $\lambda$. 
Proof 1. Consider the standard inner product on $L^{2}(0, \infty ; \mathbb{C})$, namely

$$
\langle f, g\rangle_{L^{2}(0, \infty ; \mathbb{C})}=\int_{0}^{\infty} f(t) \bar{g}(t) d t \quad \forall f, g \in L^{2}(0, \infty ; \mathbb{C}) .
$$

Using (45) and (27) we may write

$$
\int_{0}^{\infty} \mathcal{T}_{11}(t) b u(t) d t=b\left\langle\mathcal{T}_{11}, \bar{u}\right\rangle_{L^{2}(0, \infty ; \mathbb{C})}
$$

assuming that $\mathcal{T}_{11} \in L^{2}(0, \infty ; \mathbb{C})$. This assumption is equivalent, due to the Paley-Wiener Theorem 2.1, to $\mathcal{L}\left(\mathcal{T}_{11}\right) \in H^{2}\left(\mathbb{C}_{+}\right)$, where the last inclusion holds. Indeed, using (46) and (47) we see that $\mathcal{L}\left(\mathcal{T}_{11}\right)(s)=b R_{11}(s)=\frac{b}{s-\lambda \mathrm{e}^{-s \tau}}$. Now the assumption on $\lambda$ gives $R_{11} \in H^{2}\left(\mathbb{C}_{+}\right)$and the result follows.

2. The boundary trace $R_{11}^{*}=\mathcal{L}\left(\mathcal{T}_{11}\right)^{*} \in L^{2}(i \mathbb{R})$ is given a.e. as

$$
\mathcal{L}\left(\mathcal{T}_{11}\right)^{*}(i \omega)=\frac{1}{i \omega-\lambda \mathrm{e}^{-i \omega \tau}}
$$

Again by Theorem 2.1 and definition of the inner product on $H^{2}(\mathbb{C})_{+}$in (4) we have

$$
\begin{gathered}
b\left\langle\mathcal{T}_{11}, \bar{u}\right\rangle_{L^{2}(0, \infty ; \mathbb{C})}=b\left\langle\mathcal{L}\left(\mathcal{T}_{11}\right)^{*}, \mathcal{L}(\bar{u})^{*}\right\rangle_{L^{2}(i \mathbb{R})} \\
=\frac{b}{2 \pi} \int_{-\infty}^{+\infty} \frac{1}{i \omega-\lambda \mathrm{e}^{-i \omega \tau}} \overline{\mathcal{L}(\bar{u})^{*}}(i \omega) d \omega
\end{gathered}
$$

The Cauchy-Schwarz inequality now gives

$$
\begin{aligned}
& |b|\left|\frac{1}{2 \pi} \int_{-\infty}^{+\infty} \frac{1}{i \omega-\lambda \mathrm{e}^{-i \omega \tau}} \overline{\mathcal{L}(\bar{u})^{*}}(i \omega) d \omega\right| \\
& \leq|b|\left(\frac{1}{2 \pi} \int_{-\infty}^{+\infty}\left|\frac{1}{i \omega-\lambda \mathrm{e}^{-i \omega \tau}}\right|^{2} d \omega\right)^{\frac{1}{2}}\left(\frac{1}{2 \pi} \int_{-\infty}^{+\infty}\left|\overline{\mathcal{L}(\bar{u})^{*}}(i \omega)\right|^{2} d \omega\right)^{\frac{1}{2}} \\
& \quad=|b|\left(\frac{1}{2 \pi} \int_{-\infty}^{+\infty}\left|\frac{1}{i \omega-\lambda \mathrm{e}^{-i \omega \tau}}\right|^{2} d \omega\right)^{\frac{1}{2}}\|u\|_{L^{2}(0, \infty ; \mathbb{C})}
\end{aligned}
$$

Combining this result with point 1 we obtain

$$
\left|\int_{0}^{\infty} \mathcal{T}_{11}(t) b u(t) d t\right|^{2} \leq|b|^{2}\left(\frac{1}{2 \pi} \int_{-\infty}^{+\infty}\left|\frac{1}{i \omega-\lambda \mathrm{e}^{-i \omega \tau}}\right|^{2} d \omega\right)\|u\|_{L^{2}(0, \infty ; \mathbb{C})}^{2} .
$$

3. Consider now the second element of the forcing operator (45), namely

$$
\int_{0}^{\infty} \mathcal{T}_{21}(t) b u(t) d t \in W^{-1,2}(-\tau, 0 ; \mathbb{C})
$$


To shorten the notation we write $W:=W^{-1,2}(-\tau, 0 ; \mathbb{C})$. If we assume that $\mathcal{T}_{21} \in L^{2}(0, \infty ; W)$ then using the vector-valued version of Theorem 2.1 this is equivalent to $\mathcal{L}\left(\mathcal{T}_{21}\right) \in H^{2}\left(\mathbb{C}_{+}, W\right)$, but the last inclusion holds. Indeed, to show it notice that

$$
\epsilon_{s}(\sigma):=\mathrm{e}^{s \sigma}, \quad \sigma \in[-\tau, 0]
$$

is, as a function of $s$, analytic everywhere for every value of $\sigma$, and follow exactly the reasoning in point 1 .

4. We introduce an auxiliary function $\phi:[0, \infty) \rightarrow \mathbb{C}$. For that purpose fix $\mathcal{T}_{21} \in$ $L^{2}(0, \infty ; W)$ and $x_{0} \in W$ and define

$$
\phi(t):=\left\langle\mathcal{T}_{21}(t), x_{0}\right\rangle_{W}
$$

The Cauchy-Schwarz inequality gives

$$
\int_{0}^{\infty}\left|\left\langle\mathcal{T}_{21}(t), x_{0}\right\rangle_{W}\right|^{2} d t \leq \int_{0}^{\infty}\left\|\mathcal{T}_{21}(t)\right\|_{W}^{2} d t\left\|x_{0}\right\|_{W}^{2}<\infty
$$

hence $\phi \in L^{2}(0, \infty ; \mathbb{C})$.

5. Consider now the following:

$b \int_{0}^{\infty} \phi(t) u(t) d t=b \int_{0}^{\infty}\left\langle\mathcal{T}_{21}(t), x_{0}\right\rangle_{W} u(t) d t=b\left\langle\int_{0}^{\infty} \mathcal{T}_{21}(t) u(t) d t, x_{0}\right\rangle_{W}$.

We also have

$$
b \int_{0}^{\infty} \phi(t) u(t) d t=b\langle\phi, \bar{u}\rangle_{L^{2}(0, \infty ; \mathbb{C})}=b\left\langle\mathcal{L}(\phi)^{*}, \mathcal{L}(\bar{u})^{*}\right\rangle_{L^{2}(i \mathbb{R})} .
$$

To obtain the boundary trace $\mathcal{L}(\phi)^{*}$ notice that

$$
\begin{aligned}
\mathcal{L}(\phi)(s) & =\int_{0}^{\infty} \mathrm{e}^{-s r}\left\langle\mathcal{T}_{21}(r), x_{0}\right\rangle_{W} d r=\left\langle\int_{0}^{\infty} \mathrm{e}^{-s r} \mathcal{T}_{21}(r) d r, x_{0}\right\rangle_{W} \\
& =\left\langle\mathcal{L}\left(\mathcal{T}_{21}\right)(s), x_{0}\right\rangle_{W}=\left\langle R_{21}(s), x_{0}\right\rangle_{W} .
\end{aligned}
$$

Using now (46) yields the result

$$
\mathcal{L}(\phi)^{*}(i \omega)=\left\langle R_{21}^{*}(i \omega), x_{0}\right\rangle_{W}=\left\langle\frac{\epsilon_{i \omega}}{i \omega-\lambda \mathrm{e}^{-i \omega \tau}}, x_{0}\right\rangle_{W}
$$

Finally, using the inner product on $L^{2}(i \mathbb{R})$ and the fact that $\overline{\mathcal{L}(\bar{u})} *(i \omega) \in \mathbb{C}$ for every $\omega \in \mathbb{R}$ we obtain

$$
\left\langle\int_{0}^{\infty} \mathcal{T}_{21}(t) u(t) d t, x_{0}\right\rangle_{W}=\left\langle\frac{1}{2 \pi} \int_{-\infty}^{+\infty} R_{21}^{*}(i \omega) \overline{\mathcal{L}(\bar{u})^{*}}(i \omega) d \omega, x_{0}\right\rangle_{W}
$$


and

$$
\int_{0}^{\infty} \mathcal{T}_{21}(t) u(t) d t=\frac{1}{2 \pi} \int_{-\infty}^{+\infty} R_{21}^{*}(i \omega) \overline{\mathcal{L}(\bar{u})} *(i \omega) d \omega \in W
$$

6. Using the norm on $L^{2}(-\tau, 0 ; \mathbb{C})$ we have

$$
\begin{aligned}
\left\|R_{21}^{*}(i \omega)\right\|_{L^{2}(-\tau, 0 ; \mathbb{C})}^{2} & =\int_{-\tau}^{0}\left|\frac{\mathrm{e}^{i \omega t}}{i \omega-\lambda \mathrm{e}^{-i \omega \tau}}\right|^{2} d t=\frac{1}{\left|i \omega-\lambda \mathrm{e}^{-i \omega \tau}\right|^{2}} \int_{-\tau}^{0}\left|\mathrm{e}^{i \omega t}\right|^{2} d t \\
& =\frac{\tau}{\left|i \omega-\lambda \mathrm{e}^{-i \omega \tau}\right|^{2}} .
\end{aligned}
$$

The Cauchy-Schwarz inequality gives

$$
\begin{aligned}
& |b|\left\|\frac{1}{2 \pi} \int_{-\infty}^{+\infty} R_{21}^{*}(i \omega) \overline{\mathcal{L}(\bar{u})^{*}}(i \omega) d \omega\right\|_{L^{2}(-\tau, 0 ; \mathbb{C})} \\
& \quad \leq|b| \frac{1}{2 \pi} \int_{-\infty}^{+\infty}\left\|R_{21}^{*}(i \omega)\right\|_{L^{2}(-\tau, 0 ; \mathbb{C})} \mid \overline{\mathcal{L}(\bar{u})^{*}(i \omega) \mid d \omega} \\
& \quad=|b| \frac{1}{2 \pi} \int_{-\infty}^{+\infty} \frac{\tau^{\frac{1}{2}}}{\left|i \omega-\lambda \mathrm{e}^{-i \omega \tau}\right|}\left|\overline{\mathcal{L}(\bar{u})^{*}}(i \omega)\right| d \omega \\
& \quad \leq|b|\left(\frac{1}{2 \pi} \int_{-\infty}^{+\infty}\left(\frac{\tau^{\frac{1}{2}}}{\left|i \omega-\lambda \mathrm{e}^{-i \omega \tau}\right|}\right)^{2} d \omega\right)^{\frac{1}{2}}\left(\frac{1}{2 \pi} \int_{-\infty}^{+\infty} \mid \overline{\left.\left.\mathcal{L}(\bar{u})^{*}(i \omega)\right|^{2} d \omega\right)^{\frac{1}{2}}}\right. \\
& \quad=|b|\left(\frac{1}{2 \pi} \int_{-\infty}^{+\infty} \frac{\tau}{\left|i \omega-\lambda \mathrm{e}^{-i \omega \tau}\right|^{2}} d \omega\right)^{\frac{1}{2}}\|u\|_{L^{2}(0, \infty ; \mathbb{C})}
\end{aligned}
$$

Combining this result with point 5 gives

$$
\left\|\int_{0}^{\infty} \mathcal{T}_{21}(t) b u(t) d t\right\|_{L^{2}(-\tau, 0 ; \mathbb{C})} \leq|b|\left(\frac{1}{2 \pi} \int_{-\infty}^{+\infty} \frac{\tau}{\left|i \omega-\lambda \mathrm{e}^{-i \omega \tau}\right|^{2}} d \omega\right)^{\frac{1}{2}}\|u\|_{L^{2}(0, \infty ; \mathbb{C})}
$$

7. Taking now the norm $\|\cdot\| \mathcal{X}$ resulting from (23) and using (45), (49) and (51) we arrive at

$$
\begin{aligned}
\left\|\Phi_{\infty}(u)\right\|_{\mathcal{X}}^{2} & =\left|\int_{0}^{\infty} \mathcal{T}_{11}(t) b u(t) d t\right|^{2}+\left\|\int_{0}^{\infty} \mathcal{T}_{21}(t) b u(t) d t\right\|_{L^{2}(-\tau, 0 ; \mathbb{C})}^{2} \\
& \leq|b|^{2}(1+\tau)\left(\frac{1}{2 \pi} \int_{-\infty}^{+\infty} \frac{1}{\left|i \omega-\lambda \mathrm{e}^{-i \omega \tau}\right|^{2}} d \omega\right)\|u\|_{L^{2}(0, \infty ; \mathbb{C})}^{2}
\end{aligned}
$$

The remaining part is to deal with the integral in the above estimation. Note, that trying to calculate it directly this problem is equivalent (up to a constant) to calculation of the integral 


$$
\int_{-i \infty}^{+i \infty} \frac{d s}{\left(s-\lambda \mathrm{e}^{-s \tau}\right)\left(-s-\bar{\lambda} \mathrm{e}^{s \tau}\right)}
$$

with $s$ and $\lambda$ as complex variables. This inevitably leads to the Lambert-W function related pole placement and complications with finding a suitable contour of integration. To avoid these difficulties we will content ourselves with estimation only.

8. Define

$$
\int_{-\infty}^{+\infty} I(i \omega) d \omega:=\int_{-\infty}^{+\infty} \frac{1}{\left|i \omega-\lambda \mathrm{e}^{-i \omega \tau}\right|^{2}} d \omega .
$$

From the fact that for $\omega \in \mathbb{R}$ there is

$$
\left|i \omega-\lambda \mathrm{e}^{-i \omega \tau}\right|=\left|-i \omega-\bar{\lambda} \mathrm{e}^{i \omega \tau}\right|,
$$

the equalities

$$
\begin{aligned}
\int_{0}^{+\infty} \frac{1}{\left|i \omega-\lambda \mathrm{e}^{-i \omega \tau}\right|^{2}} d \omega & =\int_{-\infty}^{0} \frac{1}{\left|i \omega-\bar{\lambda} \mathrm{e}^{-i \omega \tau}\right|^{2}} d \omega, \\
\int_{-\infty}^{0} \frac{1}{\left|i \omega-\lambda \mathrm{e}^{-i \omega \tau}\right|^{2}} d \omega & =\int_{0}^{+\infty} \frac{1}{\left|i \omega-\bar{\lambda} \mathrm{e}^{-i \omega \tau}\right|^{2}} d \omega
\end{aligned}
$$

follow. They give

$$
\int_{-\infty}^{+\infty} \frac{1}{\left|i \omega-\lambda \mathrm{e}^{-i \omega \tau}\right|^{2}} d \omega=\int_{-\infty}^{+\infty} \frac{1}{\left|i \omega-\bar{\lambda} \mathrm{e}^{-i \omega \tau}\right|^{2}} d \omega
$$

and therefore one can consider the sole case $\operatorname{Arg}(\lambda) \in\left(\frac{\pi}{2}, \pi\right]$. Using the reverse triangle inequality we may now write

$$
\begin{aligned}
\int_{-\infty}^{+\infty} I(i \omega) d \omega= & \int_{-\infty}^{0} \frac{1}{\left|i \omega-\lambda \mathrm{e}^{-i \omega \tau}\right|^{2}} d \omega+\int_{0}^{+\infty} \frac{1}{\left|i \omega-\lambda \mathrm{e}^{-i \omega \tau}\right|^{2}} d \omega \\
= & \int_{0}^{+\infty} \frac{1}{\left|i \omega-\bar{\lambda} \mathrm{e}^{-i \omega \tau}\right|^{2}} d \omega+\int_{0}^{+\infty} \frac{1}{\left|i \omega-\lambda \mathrm{e}^{-i \omega \tau}\right|^{2}} d \omega \\
\leq & \int_{0}^{(1-\delta)|\bar{\lambda}|} \frac{d \omega}{(|\omega|-|\bar{\lambda}|)^{2}}+\int_{(1+\delta)|\bar{\lambda}|}^{+\infty} \frac{d \omega}{(|\omega|-|\bar{\lambda}|)^{2}} \\
& +\int_{(1-\delta)|\bar{\lambda}|}^{(1+\delta)|\bar{\lambda}|} \frac{1}{\left|i \omega-\bar{\lambda} \mathrm{e}^{-i \omega \tau}\right|^{2}} d \omega \\
& +\int_{0}^{(1-\delta)|\lambda|} \frac{d \omega}{(|\omega|-|\lambda|)^{2}}+\int_{(1+\delta)|\lambda|}^{+\infty} \frac{d \omega}{(|\omega|-|\lambda|)^{2}} \\
& +\int_{(1-\delta)|\lambda|}^{(1+\delta)|\lambda|} \frac{1}{\left|i \omega-\lambda \mathrm{e}^{-i \omega \tau}\right|^{2}} d \omega
\end{aligned}
$$




$$
\begin{aligned}
= & 2 \int_{0}^{(1-\delta)|\lambda|} \frac{d \omega}{(\omega-|\lambda|)^{2}}+2 \int_{(1+\delta)|\lambda|}^{+\infty} \frac{d \omega}{(\omega-|\lambda|)^{2}} \\
& +\int_{(1-\delta)|\bar{\lambda}|}^{(1+\delta)|\bar{\lambda}|} \frac{1}{\left|i \omega-\bar{\lambda} \mathrm{e}^{-i \omega \tau}\right|^{2}} d \omega+\int_{(1-\delta)|\lambda|}^{(1+\delta)|\lambda|} \frac{1}{\left|i \omega-\lambda \mathrm{e}^{-i \omega \tau}\right|^{2}} d \omega
\end{aligned}
$$

for any $\delta \in(0,1)$. The first and second integral on the right hand side give

$$
\int_{0}^{(1-\delta)|\lambda|} \frac{d \omega}{(\omega-|\lambda|)^{2}}=\frac{1-\delta}{\delta|\lambda|}
$$

and

$$
\int_{(1+\delta)|\lambda|}^{+\infty} \frac{d \omega}{(\omega-|\lambda|)^{2}}=\frac{1}{\delta|\lambda|}
$$

Taking into account the comments above we will firstly find the upper bound for the last integral in (53).

9. Hence, using the assumption let $\lambda=|\lambda| \mathrm{e}^{i \operatorname{Arg}(\lambda)}$, where $\operatorname{Arg}(\lambda)=\frac{\pi}{2}+\varepsilon_{\lambda}$, $\varepsilon_{\lambda} \in\left(0, \frac{\pi}{2}\right]$ and

$$
0<|\lambda| \tau<\operatorname{Arg}(\lambda)-\frac{\pi}{2}=\varepsilon_{\lambda} \leq \frac{\pi}{2} .
$$

Fix now $\delta \in(0,1)$ such that

$$
(1-\delta)|\lambda| \tau<|\lambda| \tau<(1+\delta)|\lambda| \tau<\varepsilon_{\lambda}
$$

Let $\eta \in[1-\delta, 1+\delta]$ and consider $\omega=\eta|\lambda|$. For such $\omega$ we have

$$
\begin{aligned}
\left|i \omega-\lambda \mathrm{e}^{-i \omega \tau}\right|^{2} & =|\lambda|^{2}\left|\eta \mathrm{e}^{i \frac{\pi}{2}}-\mathrm{e}^{i(\operatorname{Arg}(\lambda)-\eta|\lambda| \tau)}\right|^{2} \\
& =|\lambda|^{2}\left|k_{\eta}-q_{\eta}\right|^{2}=|\lambda|^{2} v_{\eta}^{2}
\end{aligned}
$$

with the obvious definition of $k_{\eta}$ and $q_{\eta}$ vectors and $v_{\eta}:=\left|k_{\eta}-q_{\eta}\right|$. Due to (54) and the definition of $\eta$ we have

$$
\operatorname{Arg}(\lambda)-\eta|\lambda| \tau=\frac{\pi}{2}+\varepsilon_{\lambda}-\eta|\lambda| \tau>\frac{\pi}{2}
$$

and $\left|k_{\eta}-q_{\eta}\right|^{2}>0$ for every $\eta \in[1-\delta, 1+\delta]$. Define $\varepsilon(\eta)$ as the angle between $k_{\eta}$ and $q_{\eta}$, that is

$$
\varepsilon(\eta):=\varepsilon_{\lambda}-\eta|\lambda| \tau
$$


which is a linear function of $\eta \in[1-\delta, 1+\delta]$ with values

$$
\varepsilon(\eta) \in\left(\varepsilon_{\lambda}-(1+\delta)|\lambda| \tau, \varepsilon_{\lambda}-(1-\delta)|\lambda| \tau\right) \subset\left(0, \frac{\pi}{2}\right) .
$$

The law of cosines in the $\eta$-dependent triangle $\left(k_{\eta}, q_{\eta}, v_{\eta}\right)$ gives

$$
v_{\eta}^{2}=1+\eta^{2}-2 \eta \cos \left(\varepsilon_{\lambda}-\eta|\lambda| \tau\right)
$$

The strict monotonicity of the cosine function on $\left(0, \frac{\pi}{2}\right)$ and $(56)$ give

$$
m:=\max \left\{\cos \left(\varepsilon_{\lambda}-\eta|\lambda| \tau\right): \eta \in[1-\delta, 1+\delta]\right\}=\cos \left(\varepsilon_{\lambda}-(1+\delta)|\lambda| \tau\right)
$$

and $m \in(0,1)$. Hence, for every $\eta \in[1-\delta, 1+\delta]$ we have

$$
v_{\eta}^{2} \geq 1+\eta^{2}-2 \eta m \geq 1-m^{2}>0,
$$

as $\eta^{2}-2 \eta m+m^{2}=(\eta-m)^{2} \geq 0$ so that $\eta^{2}-2 \eta m \geq-m^{2}$. Now (55) and (59) give

$$
\frac{1}{\left|i \omega-\lambda \mathrm{e}^{-i \omega \tau}\right|^{2}} \leq \frac{1}{\left(1-m^{2}\right)|\lambda|^{2}} \quad \forall \omega \in[(1-\delta)|\lambda|,(1+\delta)|\lambda|]
$$

and, in consequence, lead to a finite upper bound of the last integral in (53), that is

$$
\int_{(1-\delta)|\lambda|}^{(1+\delta)|\lambda|} \frac{1}{\left|i \omega-\lambda \mathrm{e}^{-i \omega \tau}\right|^{2}} d \omega \leq \frac{2 \delta}{\left(1-m^{2}\right)|\lambda|} .
$$

Noting that $\operatorname{Arg}(\bar{\lambda})=-\operatorname{Arg}(\lambda)$ and using the same geometrical approach one can show that for the third integral in (53) the upper bound of (60) also holds.

10. Taking together (52), (53) and (60) we arrive at

$$
\left\|\Phi_{\infty}(u)\right\|_{\mathcal{X}}^{2} \leq|b|^{2}(1+\tau) \frac{1}{\pi}\left(\frac{2-\delta}{\delta|\lambda|}+\frac{2 \delta}{\left(1-m^{2}\right)|\lambda|}\right)\|u\|_{L^{2}(0, \infty ; \mathbb{C})}^{2}
$$

\subsection{Analysis of the Whole System}

Let us return now to the diagonal non-autonomous system (16) with state space $X=$ $l^{2}(\mathbb{C})$ and to denoting its $k$-th component with the subscript. As shown in the previous subsection, Proposition 3.2 states that the system (19) describing the $k$-th component is well-posed and its mild solution is given by (30), that is $v_{k}:[0, \infty) \rightarrow \mathcal{X}$,

$$
v_{k}(t)=\left(\begin{array}{c}
z_{k}(t) \\
z_{t_{k}}
\end{array}\right)=\mathcal{T}_{k}(t) v_{k}(0)+\int_{0}^{t} \mathcal{T}_{k}(t-s) \mathcal{B}_{k} u(s) d s .
$$


Given the structure of the Hilbert space $\mathcal{X}$ in (5) the mild solution (61) has values in the subspace of $\mathcal{X}$ spanned by the $k$-th element of its basis. Hence, defining $v$ : $[0, \infty) \rightarrow \mathcal{X}$

$$
v(t):=\sum_{k \in \mathbb{N}} v_{k}(t),
$$

we obtain a unique mild solution of (16) and this system is well-posed. Using (62) and (5) we have

$$
\begin{aligned}
\|v(t)\|_{\mathcal{X}}^{2} & =\left\|\left(\begin{array}{c}
z(t) \\
z_{t}
\end{array}\right)\right\|_{\mathcal{X}}^{2}=\|z(t)\|_{l^{2}}^{2}+\left\|z_{t}\right\|_{L^{2}\left(-\tau, 0 ; l^{2}\right)}^{2} \\
& =\sum_{k \in \mathbb{N}}\left|z_{k}(t)\right|^{2}+\sum_{k \in \mathbb{N}}\left|\left\langle z_{t}, l_{k}\right\rangle_{L^{2}\left(-\tau, 0 ; l^{2}\right)}\right|^{2} \\
& =\sum_{k \in \mathbb{N}}\left(\left|z_{k}(t)\right|^{2}+\left\|z_{t_{k}}\right\|_{L^{2}(-\tau, 0 ; \mathbb{C})}^{2}\right) \\
& =\sum_{k \in \mathbb{N}}\left\|v_{k}(t)\right\|_{\mathcal{X}}^{2},
\end{aligned}
$$

where we used again (22) and notation from (19). We can formally write the mild solution (62) as a function $v:[0, \infty) \rightarrow \mathcal{X}_{-1}$,

$$
v(t)=\mathcal{T}(t) v(0)+\int_{0}^{t} \mathcal{T}(t-s) \mathcal{B} u(s) d s .
$$

where $\mathcal{X}_{-1}=X_{-1} \times W^{-1,2}(-\tau, 0 ; X)$ and the control operator $\mathcal{B} \in \mathcal{L}\left(\mathbb{C}, \mathcal{X}_{-1}\right)$ is given by $\mathcal{B}=\left(\begin{array}{c}\left(b_{k}\right)_{k \in \mathbb{N}} \\ 0\end{array}\right)$. We may now state the main theorem of this article.

Theorem 3.7 Let for the given delay $\tau$ every element of the sequence $\left(\lambda_{k}\right)_{k \in \mathbb{N}}$ satisfy $\lambda_{k} \in \Lambda_{\tau}$, where $\Lambda_{\tau}$ was defined in (37). Then the control operator $\mathcal{B} \in \mathcal{L}\left(\mathbb{C}, \mathcal{X}_{-1}\right)$ given by $\mathcal{B}=\left(\begin{array}{c}\left(b_{k}\right)_{k \in \mathbb{N}} \\ 0\end{array}\right)$ is infinite-time admissible if the sequence $\left(C_{k}\right)_{k \in \mathbb{N}} \in l^{1}$, where

$$
C_{k}:=\left|b_{k}\right|^{2}(1+\tau) \frac{1}{\pi}\left(\frac{2-\delta_{k}}{\delta_{k}\left|\lambda_{k}\right|}+\frac{2 \delta_{k}}{\left(1-m_{k}^{2}\right)\left|\lambda_{k}\right|}\right)
$$

and $\delta_{k}, m_{k}$ fulfil the conditions (54) and (58).

Proof Define the forcing operator for $(64)$ as $\Phi_{\infty}: L^{2}(0, \infty) \rightarrow \mathcal{X}_{-1}$,

$$
\Phi_{\infty}(u):=\int_{0}^{\infty} \mathcal{T}(t) \mathcal{B} u(t) d t .
$$

From (62) it can be represented as

$$
\Phi_{\infty}(u)=\sum_{k \in \mathbb{N}} \Phi_{\infty_{k}}(u),
$$


where $\Phi_{\infty_{k}}(u)$ is given by (44) for every $k \in \mathbb{N}$. Then, similarly as in (63) and using the assumption we see that

$$
\left\|\Phi_{\infty}(u)\right\|_{\mathcal{X}}^{2}=\sum_{k \in \mathbb{N}}\left\|\Phi_{\infty_{k}}(u)\right\|_{\mathcal{X}}^{2} \leq\left(\sum_{k \in \mathbb{N}}\left|C_{k}\right|\right)\|u\|_{L^{2}(0, \infty ; \mathbb{C})}^{2}<\infty
$$

\section{Examples}

In construction of an appropriate example fulfilling assumptions of Theorem 3.7 the biggest difficulty lies in the condition imposed on the eigenvalues $\left(\lambda_{k}\right)_{k \in \mathbb{N}}$ of the generator $(A, D(A))$, defined in (18). Apart from a somewhat artificial case where one could simply define $\lambda_{k}:=\left(-\frac{\pi}{2 \tau}+\varepsilon\right) \frac{1}{k}$ for some fixed $\tau, \varepsilon>0$ and all $k \in \mathbb{N}$, we provide two additional, more illustrative examples.

\subsection{Multiplication Operator}

Consider the multiplication operator on the space $L^{2}(\Omega, \mu)$, with a $\sigma$-finite measure space $(\Omega, \mathfrak{M}, \mu)$, as shown and described in detail in [6, Section I.4.b]. More precisely, for a measurable function (called symbol) $q: \Omega \rightarrow \mathbb{C}$, we call the set

$$
q_{\text {ess }}(\Omega):=\{\lambda \in \mathbb{C}: \mu(\{s \in \Omega:|q(s)-\lambda|<\varepsilon\}) \neq 0 \text { for all } \varepsilon>0\}
$$

the essential range of $q$ and define the associated multiplication operator $M_{q}$ as

$$
M_{q} f:=q \cdot f, \quad D\left(M_{q}\right):=\left\{f \in L^{2}(\Omega, \mu): q \cdot f \in L^{2}(\Omega, \mu)\right\} .
$$

The importance of this example lies in the fact that each normal operator on a Hilbert space is unitarily equivalent to a multiplication operator on some $L^{2}$ space.

From the perspective of Theorem 3.7 the multiplication operator has a useful property, namely the spectrum of $M_{q}$ is the essential range of $q$, that is

$$
\sigma\left(M_{q}\right)=q_{e s s}(\Omega) .
$$

Hence, by choosing a suitable symbol it would be easy to control the eigenvalues. However, due to the boundedness of the region of interest in Theorem 3.7, the symbol $q$ would have to be essentailly bounded, what is a neccessary and sufficient condition for the boundedness of the multiplication operator $M_{q}$ and would limit further considerations to uniformly bounded semigroups. 


\subsection{Reciprocal System}

Following [4] we introduce the notion of a state linear system $\Sigma(A, B, C, D)$ considered on the extrapolation space $X_{-1}$, where $B \in \mathcal{L}\left(U, X_{-1}\right), C \in \mathcal{L}\left(X_{1}, Y\right)$, $D \in \mathcal{L}(U, Y), A$ generates the semigroup $(T(t))$ on $X_{-1}$ and $G$ is a transfer function of this system.

Suppose that system $\Sigma(A, B, C, D)$ is such that $0 \in \rho(A)$. Then its reciprocal system is the state linear system $\Sigma\left(A^{-1}, A^{-1} B,-C A^{-1}, G(0)\right)$. This means that for a diagonal generator $A$ with eigenvalues $\left(\lambda_{k}\right)_{k \in \mathbb{N}}$ the generator $A^{-1}$ of the reciprocal system has eigenvalues $\left(\lambda_{k}^{-1}\right)_{k \in \mathbb{N}}$.

Note that by Theorem 5 of [4], the operator $B$ is admissible for the semigroup $(T(t))$ if and only if $A^{-1} B$ is admissible for the reciprocal semigroup generated by $A^{-1}$.

Consider a heat propagation model in a homogeneous rod with zero temperature imposed on its both ends (see [21, Example 2.6.9] for more details). In terms of PDEs this model takes the form

$$
\left\{\begin{array}{lr}
\frac{\partial w}{\partial t}(x, t)=\frac{\partial^{2} w}{\partial x^{2}}(x, t), & x \in(0, \pi), t \geq 0, \\
w(0, t)=0, w(\pi, t)=0, & t \in[0, \infty), \\
w(x, 0)=w_{0}(x), & x \in(0, \pi),
\end{array}\right.
$$

where the temperature profile belongs to the state space $X=L^{2}(0, \pi)$, the initial condition (the initial temperature distribution) is $w_{0} \in W^{2,2}(0, \pi) \cap W_{0}^{1,2}(0, \pi)$.

Define

$$
A z:=\frac{d^{2} z}{d x^{2}}, \quad D(A):=W^{2,2}(0, \pi) \cap W_{0}^{1,2}(0, \pi),
$$

and reformulate (65) into an abstract setting

$$
\dot{z}(t)=A z(t), \quad z(0)=w_{0}
$$

Note also that $0 \in \rho(A)$. For $k \in \mathbb{N}$ let $\phi_{k} \in D(A), \phi_{k}(x):=\sqrt{\frac{2}{\pi}} \sin (k x)$ for every $x \in(0, \pi)$. Then $\left(\phi_{k}\right)_{k \in \mathbb{N}}$ is an orthornormal Riesz basis in $X$ and

$$
A \phi_{k}=-k^{2} \phi_{k} \quad \forall k \in \mathbb{N}
$$

Using standard Hilbert space methods and transforming system (66) into the $l^{2}$ space (we use the same notation for the $l^{2}$ version of (66)) we see that the associated eigenvalue sequence $\left(\lambda_{k}\right)_{k \in \mathbb{N}}$ is $\lambda_{k}=-k^{2}$ for every $k \in \mathbb{N}$.

Take the delay $\tau=1$. Then the system being the reciprocal of (66) has a generator with a sequence of eigenvalues $\left(-\frac{1}{k^{2}}\right)_{k \in \mathbb{N}}$ fulfilling the assumption of Theorem 3.7. 


\section{Conclusions}

We have cast our results in the language of infinite-time admissibility, since this allowed us to make use of Laplace transform techniques, but since for exponentially stable systems (with $\sup \operatorname{Re} \lambda_{k}<0$ ) this is equivalent to finite-time admissibility, similar conclusions hold in this situation as in our main theorem, Theorem 3.7.

The region $\Lambda_{\tau}$ is a very natural one to find in our analysis, as may be seen by observing that the system with transfer function $1 /\left(s+\lambda e^{-s \tau}\right)$ (where $\tau>0$ and $\lambda \in$ $\mathbb{C}$ ) is $H^{\infty}$ stable if and only if $\lambda \in \Lambda_{\tau}$. Thus, paradoxically, a large negative eigenvalue $\lambda$, although seemingly contributing to stability, actually causes destabilization, and loss of admissibility, in the presence of delays. Thus for a system such as the heat equation, where the set of eigenvalues is not contained in any single $\Lambda_{\tau}$, one cannot expect a positive result in the presence of delay.

This is also interesting from the reciprocal systems point of view, as given in Example 4.2, for the following reason. According to [4, Theorem 5], $B$ is an infinitetime admissible operator if and only if $A^{-1} B$ is. As our analysis shows, adding a positive delay breaks this symmetry.

The last conclusion concerns the open question we formulated in [26], where we looked for admissibility criteria of retarded delay systems formed by contraction semigroups. In light of our results for diagonal state-delayed system it seems that contraction is not a sufficient condition for admissibility of a diagonal retarded delay system. Instead, sufficiency is reached when the sequence of eigenvalues of the undelayed semigroup fulfils a condition similar to $\lambda_{k} \in \Lambda_{\tau}$.

Acknowledgements This project has received funding from the European Union's Horizon 2020 research and innovation programme under the Marie Skłodowska-Curie Grant Agreement No. 700833.

Open Access This article is distributed under the terms of the Creative Commons Attribution 4.0 International License (http://creativecommons.org/licenses/by/4.0/), which permits unrestricted use, distribution, and reproduction in any medium, provided you give appropriate credit to the original author(s) and the source, provide a link to the Creative Commons license, and indicate if changes were made.

\section{References}

1. Arendt, W., Batty, C.J.K., Hieber, M., Neubrander, F.: Vector-Valued Laplace Transforms and Cauchy Problems, Monographs in Mathematics, vol. 96, 2nd edn. Birkhäuser Verlag AG, Basel (2010)

2. Batkái, A., Piazzera, S.: Semigroups for Delay Equations, Research Notes in Mathematics, vol. 10. CRC Press, Boca Raton (2005)

3. Bellman, R., Cooke, K.L.: Differential-Difference Equations, Mathematics in Science and Engineering, vol. 6. Academic Press, London (1963)

4. Curtain, R.F.: Regular linear systems and their reciprocals: applications to Riccati equations. Syst. Control Lett. 49, 81-89 (2003)

5. Engel, K.-J.: Spectral theory and generator property for one-sided coupled operator matrices. Semigroup Forum 58(2), 267-295 (1999)

6. Engel, K.-J., Nagel, R.: One-Parameter Semigroup for Linear Evolution Equations, Graduate Texts in Mathematics, vol. 194. Springer, Berlin (2000)

7. Evans, L.C.: Partial Differential Equations, Graduate Studies in Mathematics, vol. 19. American Mathematical Society, Providence (2002) 
8. Garnett, J.: Bounded Analytic Functions, Graduate Texts in Mathematics, vol. 236. Springer, New York (2007)

9. Grabowski, P., Callier, F.M.: Admissible observation operators, semigroup criteria of admissibility. Integral Equ. Oper. Theory 25(2), 182-198 (1996)

10. Ho, L.F., Russell, D.L.: Admissible input elements for systems in Hilbert space and a Carleson measure criterion. SIAM J. Control Optim. 21, 616-640 (1983)

11. Ho, L.F., Russell, D.L.: Erratum: admissible input elements for systems in Hilbert space and a Carleson measure criterion. SIAM J. Control Optim. 21, 985-986 (1983)

12. Jacob, B., Partington, J.R.: Admissibility of control and observation operators for semigroups: a survey. In: Ball, Joseph A., Helton, JWilliam, Klaus, Martin, Rodman, Leiba (eds.) Current Trends in Operator Theory and its Applications, pp. 199-221. Birkhäuser Basel, Basel (2004)

13. Jacob, B., Partington, J.R., Pott, S.: On Laplace-Carleson embedding theorems. J. Funct. Anal. 264, 783-814 (2013)

14. Jacob, B., Partington, J.R., Pott, S.: Applications of Laplace-Carleson embeddings to admissibility and controllability. SIAM J. Control Optim. 52(2), 1299-1313 (2014)

15. Kato, T.: Perturbation Theory for Linear Operators, Classics in Mathematics. Springer, Berlin (1995). Reprint of the 1980 edition

16. Koosis, P.: Introduction to $H_{p}$ Spaces, Cambridge Tracts in Mathematics, vol. 115, 2nd edn. Cambridge University Press, Cambridge (2008)

17. Kreuter, M.: Sobolev spaces of vector-valued functions. Master's thesis, Ulm University: under the supervision of W Arendt and R Zacher (2015)

18. Partington, J.R.: An Introduction to Hankel Operators, London Mathematical Society Student Texts, vol. 13. Cambridge University Press, Cambridge (1988)

19. Partington, J.R.: Linear Operators and Linear Systems: An Analytical Apporach to Control Theory, London Mathematical Society Student Texts, vol. 60. Cambridge University Press, Cambridge (2004)

20. Rudin, W.: Real and Complex Analysis, 3rd edn. McGraw-Hill, Singapore (1987)

21. Tucsnak, M., Weiss, G.: Observation and Control for Operator Semigroups. Birkhäuser Verlag AG, Basel (2009)

22. Walton, K., Marshall, J.E.: Direct method for TDS stability analysis. IEE Proc. Control Theory Appl. 134, 101-107 (1987)

23. Weiss, G.: Admissible input elements for diagonal semigroups on $l^{2}$. Syst. Control Lett. 10, 79-82 (1988)

24. Weiss, G.: A Powerful Generalization of the Carleson Measure Theorem? Open problems in Mathematical Systems and Control Theory, Communications and Control Engineering, pp. 267-272. Springer, London (1999)

25. Wynn, A.: $\alpha$-admissibility of observation operators in discrete and continuous time. Complex Anal. Oper. Theory 4(1), 109-131 (2010)

26. Zawiski, R., Partington, J.R.: On the admissibility of retarded delay systems. arXiv e-prints (2018). arXiv: 1709.08276

Publisher's Note Springer Nature remains neutral with regard to jurisdictional claims in published maps and institutional affiliations. 